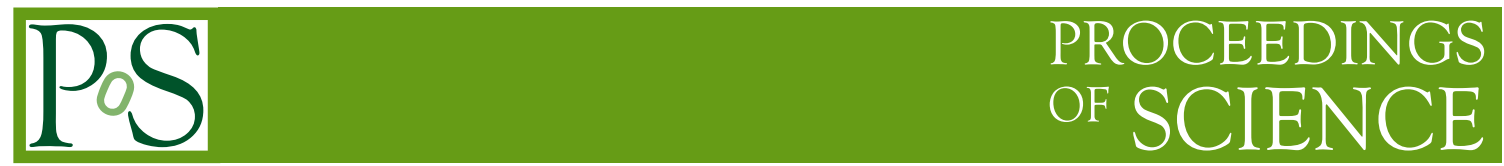

\title{
Central Exclusive Production at LHCb
}

\section{R. McNulty*}

School of Physics, University College Dublin,

Dublin 4, Ireland,

(on behalf of the LHCb collaboration.)

E-mail: ronan.mcnultyaucd.ie

Central Exclusive Production (CEP) is a unique process at hadron machines in which particles are produced via colourless propagators. LHCb have measured the cross-sections for the CEP of vector mesons, $J / \psi, \psi(2 S), \Upsilon(1 S), \Upsilon(2 S)$ and $\Upsilon(3 S)$, which are photo-produced. In the double pomeron exchange process, preliminary measurements have been made of $\chi_{c 0}, \chi_{c 1}, \chi_{c 2}$ meson production while the first observations of the CEP of pairs of charmonia, $J / \psi J / \psi$ and $J / \psi \psi(2 S)$, have been made and limits obtained on the pair production of other charmonia.

XXIV International Workshop on Deep-Inelastic Scattering and Related Subjects

11-15 April, 2016

DESY Hamburg, Germany

\footnotetext{
* Speaker.
} 


\section{Introduction}

Central exclusive production (CEP) at the LHC is characterised by an isolated system of particles surrounded by two rapidity gaps that extend down to the intact colliding protons; see [1] and the papers referenced in [2] for a recent survey of the field. The lack of additional activity signals the presence of colourless propagators: two photons; two pomerons; or a photon and a pomeron. Measurements of CEP test QCD, investigate the nature of the pomeron, and can be used to constrain the gluon PDF. At $\sqrt{s}=13 \mathrm{TeV}$, measurements of $J / \psi$ production will probe the gluon PDF down to a fractional momentum of the proton $x=2 \times 10^{-6}$, a scale at which saturation effects may become visible.

The LHCb detector [3] is suited to studying CEP as it is fully instrumented with tracking, calorimetry and particle identification in the pseudorapidity, $\eta$, range between 2 and 5. In addition, charged activity in the backward region, in the approximate range $-3.5<\eta<-1.5$, can be vetoed due to the presence of a silicon strip detector that surrounds the interaction point. LHCb is designed to trigger on particles produced at low transverse momentum. CEP events are selected by triggering on muons with transverse momentum, $p_{T}$, above $400 \mathrm{MeV} / \mathrm{c}$ or electromagnetic or hadronic energy above $1000 \mathrm{MeV}$, in coincidence with a total event charged multiplicity of less than 10 as recorded by a scintillating pad detector. A further advantage of LHCb for CEP is the low number of protonproton interactions (typically 1.5 ) per beam crossing.

The LHCb measurements of CEP to date have concentrated on final states with muons. In this report, I focus on measurements of the photoproduction of single charmonium and bottomium as well as the production of double charmonia, which is principally produced by double pomeron exchange. The single charmonium measurements use almost $1 \mathrm{fb}^{-1}$ of data taken at $\sqrt{s}=7 \mathrm{TeV}$, while the other measurements add an additional $2 \mathrm{fb}^{-1}$ at $\sqrt{s}=8 \mathrm{TeV}$. Preliminary results, based on $37 \mathrm{pb}^{-1}$ of data, are also available on $\chi_{c}$ production and dimuons produced by the QED diphoton exchange process [4]. New forward scintillators have been installed for the $\sqrt{s}=13 \mathrm{TeV}$ running, and their impact on future measurements will be briefly discussed.

\section{Photoproduction of $J / \psi$ and $\psi(2 S)$ mesons}

Candidates for $J / \psi$ mesons produced through CEP are selected [5] by requiring two identified muons inside the $\mathrm{LHCb}$ acceptance and no photons or additional tracks in either forward or backward directions. The $p_{T}^{2}$ of the dimuon is required to be below $0.8 \mathrm{GeV}^{2} / \mathrm{c}^{2}$, and its invariant mass to be within $65 \mathrm{MeV} / \mathrm{c}^{2}$ of the known $J / \psi$ or $\psi(2 S)$ masses. The invariant mass of all candidates (with the mass requirement removed) is shown in Fig.1 (left). The non-resonant background, due to the QED production of dimuons via photon propagators, is modelled with an exponential function and is estimated to account for $(0.8 \pm 0.1) \%$ of the $J / \psi$ and $(16 \pm 3) \%$ of the $\psi(2 S)$ sample. Feed-down backgrounds inside the $J / \psi$ mass window, amounting to $(10.1 \pm 0.9) \%$, are due to $\chi_{c}$ or $\psi(2 S)$ mesons decaying to $J / \psi$ and photons that are undetected due to being very soft or going outside the detector acceptance. Inelastic $J / \psi$ or $\psi(2 S)$ production, in which the proton dissociates but does not produce activity inside the LHCb acceptance, is assessed by fitting the $t \approx p_{T}^{2}$ distribution and assuming that $d \sigma / d t$ can be modelled by two exponentials for signal and background, as assumed in Regge theory and observed at HERA [6, 7]. The fitted parameters for the 
exponentials are consistent with those found at HERA, having corrected for kinematic differences. In total, $(59.2 \pm 1.2) \%$ of the $J / \psi$ sample and $(52 \pm 7) \%$ of the $\psi(2 S)$ sample is estimated to be exclusively produced.
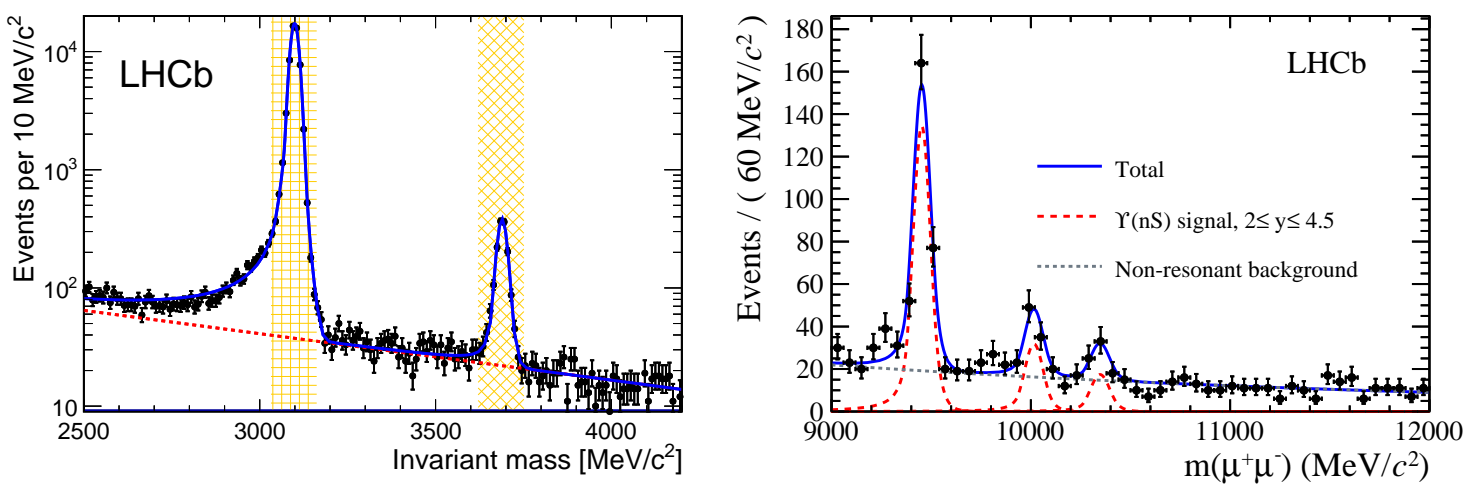

Figure 1: Invariant mass of dimuons in (left) the charmonium analysis [5] with the $J / \psi$ and $\psi(2 S)$ windows indicated and (right) the bottomonium analysis [15].

The cross-section for CEP charmonia is determined from the estimated number of CEP events, correcting for the detector efficiency and acceptance: the former is determined with tag-and-probe techniques in data while the latter is found from simulated events. The total cross-sections are given in Table 1 while differential cross-sections as a function of rapidity are shown in Fig. 2 compared to LO and approximate NLO predictions from [8]. Results on the total cross-section have been compared to other predictions $[9,10,11,12,13]$ and all agree with the data. A photoproduction cross-section can be derived from these results once rapidity gap corrections and photon flux factors are included. A two-fold ambiguity is present due to not knowing which of the protons the photon was radiated from. This can be resolved in a model-dependent way by assuming the H1 derived power-law ${ }^{1}$ for one of the solutions. The (model-dependent) cross-section derived is shown in Fig. 2, compared to results from HERA, fixed target collisions, and proton-lead collisions at ALICE [14], in which the aforementioned ambiguity can be resolved.

\section{Photoproduction of $\Upsilon$ mesons}

Candidates for $\Upsilon$ mesons produced through CEP are selected [15] by requiring two muons in LHCb and no other charged tracks. The muon pair must have $p_{T}^{2}<2 \mathrm{GeV}^{2} / \mathrm{c}^{2}$ and an invariant mass between 9 and $20 \mathrm{GeV} / \mathrm{c}^{2}$, which allows the shape of the continuum to be determined at the same time as the fit to the $\Upsilon(1 S), \Upsilon(2 S)$ and $\Upsilon(3 S)$. The distribution of candidates is shown in Fig.1 (right).

Feed-down backgrounds coming from the various $\chi_{b}$ states are estimated to contribute $39 \pm 7 \%$ of the total signal yield. As in the charmonium analysis, inelastic $\Upsilon$ production is assessed by fitting the $t \approx p_{T}^{2}$ distribution, but with the signal shape given by the SUPERCHIC generator[13]. After subtraction of the $\chi_{b}$ component, $(54 \pm 11) \%$ is assessed to be exclusively produced; thus of the total $\Upsilon$ yield, one third is CEP.

\footnotetext{
${ }^{1}$ The power-law for $J / \psi$ is taken from [6] while for $\psi(2 S)$, I use $R(W)=\sigma(\psi(2 S)) / \sigma(J / \psi)=0.166$ from [7].
} 


\begin{tabular}{|c|c|c|}
\hline Quantity measured & Kinematic region & Measurement (pb) \\
\hline$\sigma(p p \rightarrow p J / \psi p) \cdot B R(J / \psi \rightarrow \mu \mu)$ & $2<\eta_{\mu}, y_{J / \psi}<4.5$ & $291 \pm 7 \pm 19[5]$ \\
$\sigma(p p \rightarrow p \psi(2 S) p) \cdot B R(\psi(2 S) \rightarrow \mu \mu)$ & $2<\eta_{\mu}, y_{\psi(2 S)}<4.5$ & $6.5 \pm 0.9 \pm 0.4[5]$ \\
$\sigma(p p \rightarrow p \Upsilon(1 S) p)$ & $2<\eta_{\mu}, y_{\Upsilon(1 S)}<4.5$ & $9.0 \pm 2.1 \pm 1.7[15]$ \\
$\sigma(p p \rightarrow p \Upsilon(2 S) p)$ & $2<\eta_{\mu}, y_{\Upsilon(2 S)}<4.5$ & $1.3 \pm 0.8 \pm 0.3[15]$ \\
$\sigma(p p \rightarrow p \Upsilon(3 S) p)$ & $2<\eta_{\mu}, y_{\Upsilon(3 S)}<4.5$ & $<3.4$ at $95 \%$ c.l. [15] \\
$\sigma(J / \psi J / \psi)$ & $2<y_{J / \psi J / \psi}<4.5$ & $58 \pm 10 \pm 6[20]$ \\
$\sigma(J / \psi \psi(2 S))$ & $2<y_{J / \psi \psi(2 S)}<4.5$ & $63_{-18}^{+27} \pm 10[20]$ \\
$\sigma(\psi(2 S) \psi(2 S)$ & $2<y_{\psi(2 S) \psi(2 S)}<4.5$ & $<237$ at $90 \%$ c.l. [20] \\
$\sigma\left(\chi_{c 0} \chi_{c 0}\right)$ & $2<y_{\chi_{c 0} \chi_{c 0}}<4.5$ & $<69000$ at $90 \%$ c.l. [20] \\
$\sigma\left(\chi_{c 1} \chi_{c 1}\right)$ & $2<y_{\chi_{c 1} \chi_{c 1}}<4.5$ & $<45$ at $90 \%$ c.l. [20] \\
$\sigma\left(\chi_{c 2} \chi_{c 2}\right)$ & $2<y_{\chi_{c 2} \chi_{c 2}}<4.5$ & $<141$ at $90 \%$ c.l. [20] \\
\hline
\end{tabular}

Table 1: Total cross-section results for charmonia and bottomonia states. The double charmonia crosssections include events where proton dissociation occurs.

After correcting for the detection efficiency, found using simulated events, and taking account of the luminosity, the cross-sections are determined and reported in Table 1. The low statistics and sizeable background impact on the significance of the $2 \mathrm{~S}$ and $3 \mathrm{~S}$ states. However, there are sufficient statistics to divide the $1 \mathrm{~S}$ state into three bins of rapidity, which are plotted in Fig.2. Compared to the charmonium analysis, the difference between the LO and NLO predictions is large and the data show a clear preference for the latter. A photoproduction cross-section can be derived from these results. Here, the smaller of the two ambiguous solutions (that contributes between 5\% and 20\%) is ignored. The result is shown in Fig.2 showing good consistency with HERA results $[16,17]$.

\section{Production of pairs of charmonia}

The pair production of charmonia has been observed inclusively in a previous LHCb analysis [18], where tetraquark contributions or double parton scattering (DPS) may play an important role. The contribution of DPS in CEP is minimal and the process proceeds dominantly through double pomeron exchange. The cross-section for CEP of $J / \psi J / \psi$ inside the LHCb acceptance is predicted to lie in the range $2-20 \mathrm{pb}$, depending on the model used for the soft survival factor and the gluon PDF that enters with the fourth power [19].

The selection of the CEP of charmonia pairs [20] requires exactly four tracks, at least three of which are identified as muons. The invariant masses of two unlike-sign pairs of muons is required to be consistent with that of the $J / \psi$ or $\psi(2 S)$ mesons. The pairwise combinations are shown in Fig.3(a); 37 candidates are consistent with $J / \psi J / \psi$ production and 5 are consistent with $J / \psi \psi(2 S)$. The invariant mass of the four tracks is shown in Fig.3(b) and agrees qualitatively with the spectrum observed inclusively [18]. The signal is only seen when there are precisely 4 tracks in the event; it is not present when additional tracks are present in the detector. After accounting for efficiency and luminosity, the cross-section for pairs of charmonia produced in the absence of other charged or neutral activity in LHCb is determined and given in Table 1. 

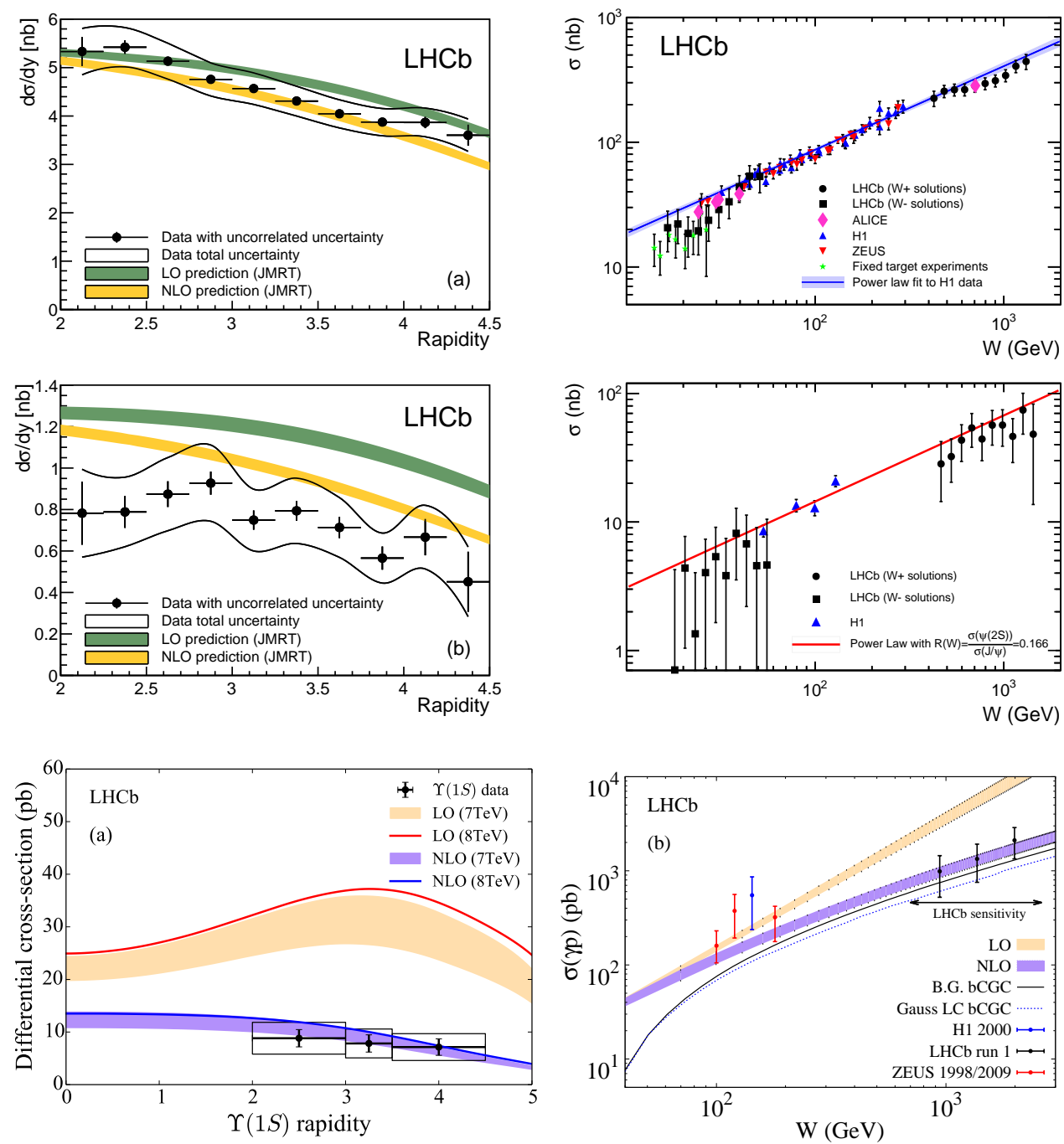

Figure 2: Differential cross-sections for exclusively produced (top left) $J / \psi$, (centre left) $\psi(2 S)$, and (bottom left) $\Upsilon$, compared to LO and NLO predictions [8]. Compilation of photoproduction cross-sections from various experiments for (top right) $J / \psi$, (centre right) $\psi(2 S)$, (bottom right) $\Upsilon$. The LHCb results are model-dependent as explained in the text.

Because of the low statistics, it is difficult to assess how often proton dissociation occurs. A fit to the $t$ distribution suggests $(42 \pm 13) \%$ of the sample is CEP implying an exclusive cross-section for $J / \psi J / \psi$ inside the $\mathrm{LHCb}$ acceptance of $(24 \pm 9) \mathrm{pb}$, in broad agreement with the predictions.

The analysis is also sensitive to the production of pairs of $\chi_{c}$ mesons, which would be visible through their decays to a $J / \psi$ meson and a photon. Searches are made for additional photons in events with two $J / \psi$ candidates. A single event is consistent with $\chi_{c 0} \chi_{c 0}$ production but is also consistent with partially reconstructed $J / \psi \psi(2 S)$ production. Limits are set on pairs of tensor meson charmonia and are given in Table 1. 

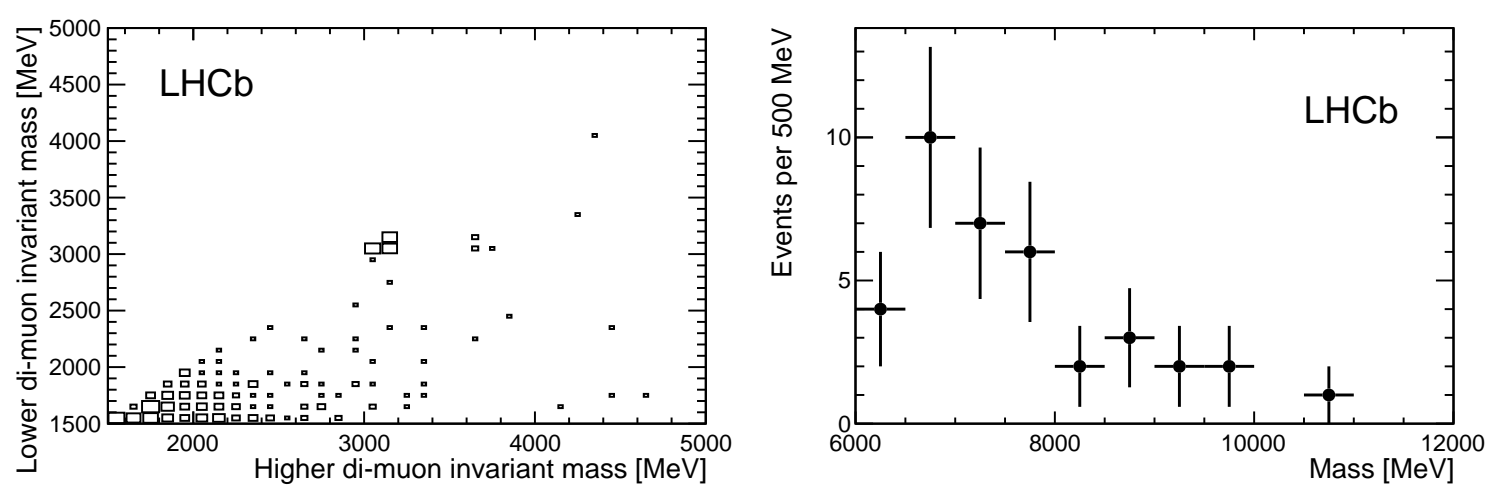

Figure 3: Invariant mass of (a, left) dimuon pairs (b, right) the $J / \psi J / \psi$ system [20].

\section{Future prospects}

Experimentally, $\mathrm{LHCb}$ is sensitive to about 5.5 units in pseudorapidity allowing modest rapidity gap sizes to be identified. The identification of CEP events would be significantly aided if the veto region could be increased. To this end, for the $\sqrt{s}=13 \mathrm{TeV}$ data-taking, forward shower counters, consisting of five planes of scintillators, have been installed perpendicular to the beam at $-114 \mathrm{~m},-19.7 \mathrm{~m},-7.5 \mathrm{~m},+20 \mathrm{~m}$ and $+114 \mathrm{~m}$ from the interaction point. With these, LHCb has sensitivity to particles in the regions $-10<\eta<-5,-3.5<\eta<-1.5,1.5<\eta<10$. The effect of the additional veto on the composition of a sample of $J / \psi$ candidates gathered at $\sqrt{s}=13 \mathrm{TeV}$ can be seen in Fig. 4. The estimated proton dissociation contribution is roughly halved in the signal region below $p_{T}^{2}=0.8 \mathrm{GeV}^{2}$, with an even greater suppression at higher values.
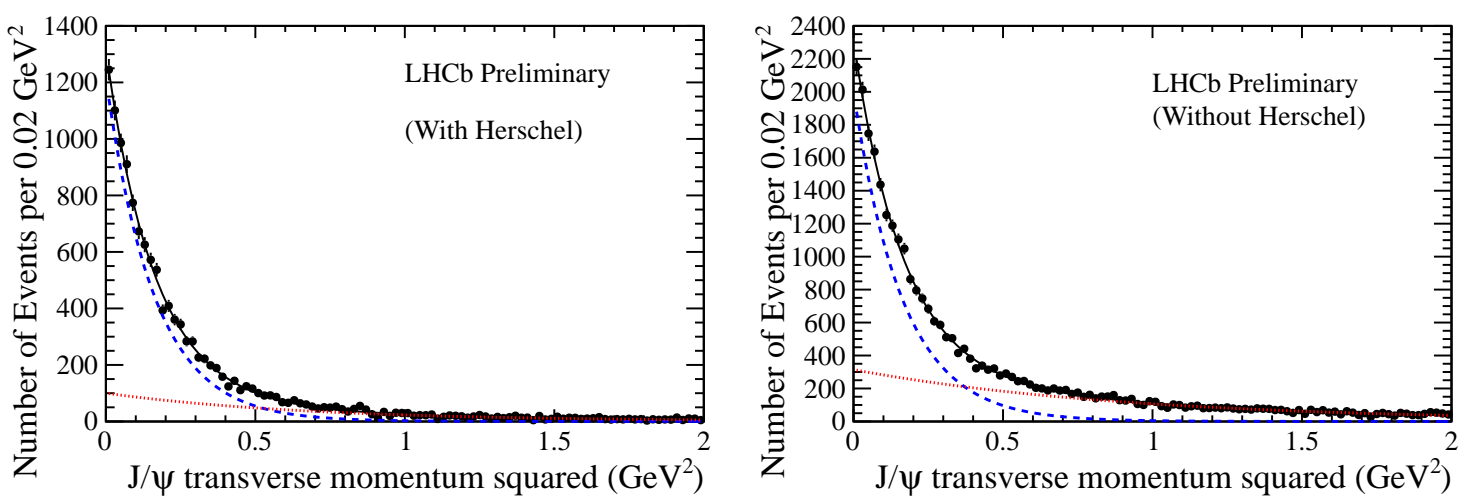

Figure 4: Transverse momentum squared of $J / \psi$ candidates at $\sqrt{s}=13 \mathrm{TeV}$ with (left) and without (right) the use of newly installed scintillators close to the beamline [21].

Additional improvements for the $\sqrt{s}=13 \mathrm{TeV}$ running include a reduction in the hadronic trigger thresholds, which will allow LHCb to extend current studies to investigate CEP of light vector mesons, a region of spectroscopic interest where the presumed dominance of the double pomeron exchange mechanism provides a gluon-rich laboratory to search for glueballs and saturation effects. 


\section{References}

[1] M.G. Albrow, T.D. Coughlin, J.R. Forshaw, Prog.Part.Nucl.Phys. 65149 (2010), arXiv:1006.1289.

[2] M.G. Albrow, Int.J.Mod. Phys. A29 1402006 (2014).

[3] LHCb collaboration, JINST 3 S08005 (2008).

[4] LHCb collaboration, CERN-LHCb-CONF-2011-022 (2011).

[5] LHCb collaboration, J.Phys. G41 055002 (2014), arXiv:1401.3288.

[6] H1 collaboration, Eur.Phys.J.C73 2466 (2013), arXiv:1304.5162.

[7] H1 collaboration, Phys.Lett. B541 251 (2002), hep-ex/0205107.

[8] S.P. Jones et al., JHEP 1311085 (2013), arXiv:1307.7099; J.Phys. G41 055009 (2014), arXiv:1312.6795.

[9] V.P. Goncalves, M.V.T. Machado, Phys. Rev. C84 011902 (2011), arXiv:1106.3036.

[10] L. Motyka, G. Watt, Phys. Rev. D78 014023 (2008), arXiv:0805.2113.

[11] W. Schafer, A. Szczurek, Phys.Rev. D76 094014 (2007), arXiv:0705.2887. [

[12] S.R. Klein, J. Nystrand, Phys.Rev.Lett. 92142003 (2004), hep-ph/0311164.

[13] L.A. Harland-Lang et al., Eur.Phys.J. C76 9 (2016), arXiv:1508.02718. [

[14] ALICE collaboration, Phys.Rev.Lett. 113232504 (2014), arXiv:1406.7819.

[15] LHCb collaboration, JHEP 1509084 (2015), arXiv:1505.08139.

[16] H1 collaboration, Phys.Lett. B483 (2000) 23, hep-ex/0003020.

[17] ZEUS collaboration, Phys.Lett. B680 4 (2009), arXiv:0903.4205.

[18] LHCb collaboration, Phys.Lett. B707 52 (2012), arXiv:1109.0963.

[19] L.A. Harland-Lang et al., J.Phys. G42 055001 (2015), arXiv:1409.4785.

[20] LHCb collaboration, J.Phys. G41 115002 (2014), arXiv:1407.5973.

[21] LHCb collaboration, LHCb-CONF-2016-007 (2016). 